\title{
Neonatal Administration Site Complication, AE
}

National Cancer Institute

\section{Source}

National Cancer Institute. Neonatal Administration Site Complication, AE. NCI Thesaurus. Code C154947.

An adverse event in a newborn characterized by local irritation or a complication at the administration site of a drug. 